\title{
Syntactic complexity in Danish L1 teaching materials
}

\section{MARTIN HAUERBERG OLSEN AND MADS POULSEN}

The purpose of this paper is to estimate the amount of demanding syntax in a sample of teaching materials used in Danish L1 classrooms from year 3 to 9 in Danish schools. We summarize three general domains of demanding syntax: less familiar (non-canonical) constructions, complexity and underspecification. All domains of demanding syntax were found across all school years, with a largely limited progression in frequency from early to later school years. Therefore, the general picture is that even younger students may encounter demanding syntax in Danish L1 teaching materials and often to an extent that does not deviate markedly from what older students encounter. An important point is, however, that demanding syntax does not necessarily have to be a problem for comprehension. Psycholinguistic studies have found that certain less familiar constructions such as passive sentences, OVS constructions, and object relatives are difficult to comprehend when their interpretation is not guided by background knowledge. In our sample, however, most examples of these constructions were supported by background knowledge. Thus, lack of knowledge of these particular constructions probably contributes very little to comprehension difficulties in reading Danish teaching materials.

KEYWORDS: reading comprehension; syntactic complexity; readability; Danish 